\title{
The application of differential geometry theory oriented to tool path generation in five-axis $\mathrm{CNC}$ machining
}

\author{
Chen Liangji, Li Huiying* \\ Tianjin Polytechnic University, Tianjin, 300387, China
}

\begin{abstract}
How to cite this paper: Chen, L., Li, H. (2019). The application of differential geometry theory oriented to tool path generation in five-axis CNC machining. Journal of Applied Mathematics and Computation, 3(6), 627-639.
\end{abstract}

DOI: $10.26855 /$ jamc.2019.12.001

*Corresponding author: Li Huiying, Tianjin Polytechnic University, Tianjin, 300387, China.

Email: lihuiying@tjpu.edu.cn

\begin{abstract}
Aiming at the problem of local curvature interference in tool path generation of five-axis CNC machining of complex free-form surface, this paper attempts to apply the theory of differential geometry to tool path generation. Based on the tangent plane at the Cutter Contacting point $(\mathrm{CC}$ point $)$, the maximum principal curvature and minimum principal curvature of the tool surface at the $\mathrm{CC}$ point were calculated, and the normal curvature model in any direction on the tool surface was established. Similarly, for the workpiece surface, the normal curvature model in any direction on the workpiece surface was established by using the theory of maximum principal direction in differential geometry. According to the above two normal curvature mathematical models, the distance between adjacent cutting positions and the distance between adjacent tool paths were calculated and deduced. At the same time, the mathematical expression to avoid local curvature interference was given to modify the tool attitude angle. The example results show that the proposed method has good effect in avoiding local curvature interference and reducing the total length of tool path. It can provide a theoretical basis for improving machining accuracy and efficiency in the field of complex surface precision machining.
\end{abstract}

\section{Keywords}

Differential geometry theory, Computer Numerically Control, Five-axis machining, Tool path generation

\section{Introduction}

Currently, the applications for precision complex surface components are expanding, and 5-axis computer numerically controlled (CNC) machining is commonly used in the precision machining of complex surfaces. Cutter location generation and planning is one of the fundamental problems in the 5-axis CNC manufacturing field. Iso-parametric tool path generation is the most common path generation method and is simple and feasible, but redundancy and deficiency problems exist in cutter locations, which impact the accuracy and efficiency of the work [1]. The other common tool path generation method is the iso-planar method [2]. This method also suffers from redundancy and deficiency problems in cutter locations, and the calculation of intersecting lines is complex. In recent years, the constant height path generation method has gradually become a topic of interest for researchers due to the need to avoid redundant cutter locations [3].

Regardless of the method used to generate the 5-axis machining tool path, the local curvature interference between the tool and machined surface must be considered. The local curvature interference is closely related to the tool shape. Three common tool types are chosen for the cutters, namely, ball-end, flat-end and torus-end cutters. The shape of the cutting edge on the ball-end cutter is simple, and thus, the calculation is simple [4-6]. This cutter was used in three-axis machining in early research. Flat-end cutters are typically more efficient than ball-end cutters, but their cutting edges are the intersection lines of the side plan and end plan and easily generate wear and tear. The flat-end cutters have a simple geometric shape, and the models for these tools are simple. Many research studies have focused on 5 -axis cutter 
path generation methods for flat-end cutting [7-9]. In document [7], the cutting shape in the direction perpendicular to the progress direction of flat-end cutters was approximately elliptical and the ellipse near the cutter contact (CC) was approximately a circle; thus, the distance between lines was calculated based on the radius of the circle. In document [8], a formula for distance between lines was proposed first by considering the cutters and the object surface as circles perpendicular to the progressing direction. Second, the cutter scan curve surface was deduced, the curvatures along the direction perpendicular to the progress direction of the curve surface and the object surface were calculated, and the distance between lines was calculated from the two curvatures. In the above methods, the calculation of the distance between lines is oriented in the direction perpendicular to the progress direction, but the explanation for why this direction was chosen is insufficient, and the formula is so simplified that it cannot reflect the actual cutting situation.

Torus-end cutters have circle edges and are more fitting to the curvature change of the machined surface, thus producing a higher quality and efficiency [10-12]. Therefore, in this paper, we focus on the cutter path generation of torus-end cutters based on the constant height method. We also study optimized control over the cutter posture to avoid local interference of the cutters. The rest of the paper will include foundation of 5-axis cutting model, local differential geometrical analysis of the CC point, calculations of the step length and the effective process bandwidth, inspection of local interference and correction of the cutter posture.

\section{Torus-end cutter and 5-axis cutting model}

As shown in Fig $1, \sum$ is the surface of the cutting edge of the torus-end cutter with radius $r_{\mathrm{f}}$. The cutter location (CL) is the centre of the bottom side of the cutter $O$. We establish the cutter coordinate system $O-x_{\mathrm{t}} y_{\mathrm{t}} z_{\mathrm{t}}$ with $O$ as the origin and the rotation the axis of the torus-end cutter $z_{\mathrm{t}}$ as the z-axis. For the coordinate vector of $O$, the CL in the object coordinate system $W-x_{\mathrm{w}} y_{\mathrm{w}} z_{\mathrm{w}}$, is $O_{\mathrm{w}}$.

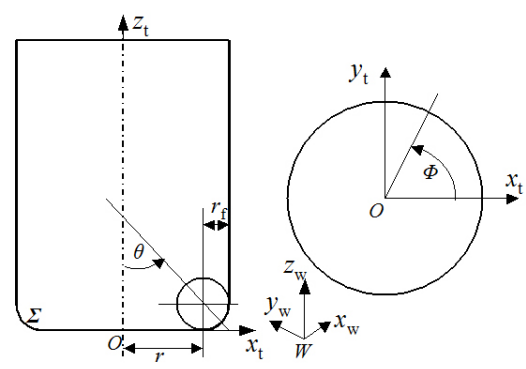

Fig 1. Torus-end cutter and cutter coordinate system

Additionally, $\sum$ is tangent to the object parameter surface $S(u, v)$ at point $C$, and these surfaces share the same tangent plane, as shown in Fig. 2, where $C$ is the CC point. The torus-end cutter moves along the $i^{\text {th }}(i=1,2, \ldots)$ CC point path $P_{i}=S(u(w), v(w))$ on surface $S$, the current CC point vector is $C=C_{i, j}=S\left(u\left(w_{j}\right), v\left(w_{j}\right)\right)$ (where $\left.j=1,2, \ldots\right)$, and the CL point vector and cutter axis unit vector are $O$ and $T$, respectively. We establish a local coordinate system $C-x_{\mathrm{c}} y_{\mathrm{c}} z_{\mathrm{c}}$ near point $C$, where the $x_{\mathrm{c}}$ axis is located along the motion direction, which is also the tangent direction of $P_{i}$ at $w=w_{j}$, the $z_{\mathrm{c}}$ axis is located along the normal direction of the surface at point $C$ (the unit normal vector is $n$ ), and the $y_{\mathrm{c}}$ axis is determined by the $x_{\mathrm{c}}$ axis and $z_{\mathrm{c}}$ axis based on the right-hand rule. We rotate along the $y_{\mathrm{c}}$ axis by $\lambda$ with the centre of the edge of the torus-end cutter as the pivot point, then rotate along the $z_{\mathrm{c}}$ axis by $\omega$ with $C$ as the pivot point, and finally reach the cutting state shown in Fig 2.

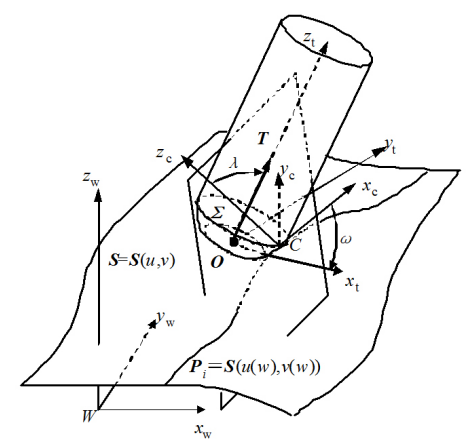

Fig 2. 5-axis cutting model for the torus-end cutter 


\section{Local differential geometrical analysis near the $\mathrm{CC}$ point}

The differential geometric character of the cutter surface and object surface at the CC point is critical for calculating the distance between lines and estimating the width of the cutting bands at the CC point. This information is also helpful for inspecting local interference and fixing the cutting location angle. The key goal of the analysis is the general expression of the normal curvature and the approach to the neighbourhood of the $\mathrm{CC}$ point. We discuss the normal curvature of the cutter surface $\Sigma$ and the object surface $S$ in the neighbourhood of the CC point $C$ and the expression of the tangent surface.

\subsection{Cutter surface}

Figure 3 shows the tangent plane at the CC point, where $P_{\Sigma 1}$ and $P_{\Sigma 2}$ are the main direction vectors corresponding to the maximum major curvature $K_{\Sigma 1}$ and minimum major curvature $K_{\Sigma 2}$, respectively, of the cutter surface $\Sigma$ at point $C$. Based on the above introduction, the angle between the direction of $P_{\Sigma 1}$ and the moving direction of the cutter is $\omega$, as shown in Fig 3.

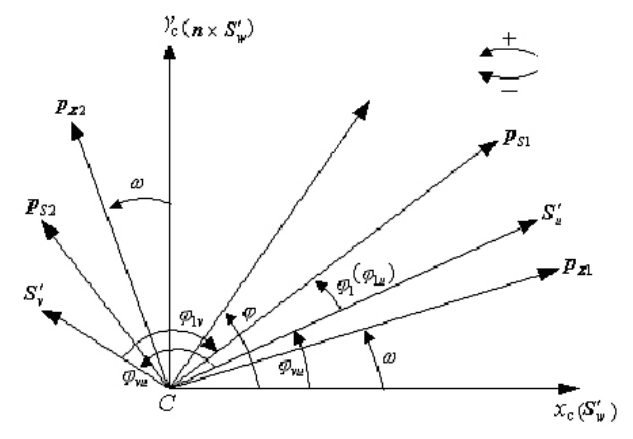

Fig 3. Tangent plane at the CC point

$K_{\Sigma 1}$ and $K_{\Sigma 2}$ can be calculated as

$$
\left\{\begin{array}{l}
K_{\Sigma 1}=\frac{1}{r_{\mathrm{f}}} \\
K_{\Sigma 2}=\frac{\sin \lambda}{r+r_{\mathrm{f}} \sin \lambda}
\end{array}\right.
$$

The normal curvature of any direction, which is angle $\varphi(-\pi / 2<\varphi \leq \pi / 2)$ from the $x_{\mathrm{c}}$ axis (moving direction of the cutter) at the point coinciding with the $\mathrm{CC}$ point in $\Sigma$, can be calculated as

$$
K_{\Sigma \varphi}=K_{\Sigma 1} \cos ^{2}(\varphi-\omega)+K_{\Sigma 2} \sin ^{2}(\varphi-\omega)
$$

If $\varphi$ is 0 and $\pm \pi / 2, K_{\Sigma 1}$ and $K_{\Sigma \pi / 2}$, the curvature along the motion direction of the cutter and its normal direction, can be obtained using the following expressions:

$$
\begin{gathered}
K_{\Sigma 0}=K_{\Sigma 1} \cos ^{2} \omega+K_{\Sigma 2} \sin ^{2} \omega \\
K_{\Sigma \pi / 2}=K_{\Sigma 1} \sin ^{2} \omega+K_{\Sigma 2} \cos ^{2} \omega
\end{gathered}
$$

By expanding Eq. (2), we obtain

$$
K_{\Sigma \varphi}=K_{\Sigma 0} \cos ^{2} \varphi+K_{\Sigma \pi / 2} \sin ^{2} \varphi+\frac{K_{\Sigma 1}-K_{\Sigma 2}}{2}+\sin 2 \varphi \sin 2 \omega
$$

We write the equation of $\Sigma^{*}$, the tangent surface of $\Sigma$, directly in the local coordinate system as

$$
\Sigma^{*}: z_{\mathrm{c}}=\frac{1}{2}\left[K_{\Sigma 0} x_{\mathrm{c}}^{2}+K_{\Sigma \pi / 2} y_{\mathrm{c}}^{2}+\left(K_{\Sigma 1}-K_{\Sigma 2}\right) \sin 2 \omega x_{\mathrm{c}} y_{\mathrm{c}}\right]
$$

\subsection{Workpiece surface}

In Fig 3, $P_{\mathrm{s} 1}$ and $P_{\mathrm{s} 2}$ are the main direction vectors corresponding to the maximum major curvature $K_{\mathrm{s} 1}$ and the minimum major curvature $K_{\mathrm{s} 2}$, respectively, of the object surface $S$ at point $C$. The direction of the two major curvatures cannot be 
determined directly, but their magnitudes can be calculated according to Ref.[8]. The next step is to determine the major directions.

In Fig 3, $S_{u}^{\prime}$ and $S_{v}^{\prime}$ represent the tangent vectors of $S$ along the $u$ and $v$ iso-perimetric lines at point $C$, respectively. The angle between $\mathrm{S}_{u}^{\prime}$ and the $x_{\mathrm{c}}$ axis (the counter-clockwise direction is positive) can be calculated as follows:

$$
\varphi_{u}=\varphi_{u}=<S_{u,}^{\prime} S_{w}^{\prime}>=\operatorname{sgn}\left(\left(S_{w}^{\prime} \times S_{u}^{\prime}\right) \cdot n\right) \arccos \frac{E u_{w}^{\prime}+E v_{w}^{\prime}}{\sqrt{E^{2} u_{w}^{\prime 2}+2 E F u_{w}^{\prime} v_{w}^{\prime}+E G v_{w}^{\prime 2}}}
$$

where $E, F$ and $G$ are type I basis quantities and $\operatorname{sgn}()$ is the function for obtaining the sign.

In Fig 3, $\varphi_{v u}$ represents the angle of $S_{v}^{\prime}$ from $S_{u}^{\prime}$ (the counter-clockwise direction is positive) and $\varphi_{v u}$ can be expressed as

$$
\varphi_{v u}=\operatorname{sgn}\left(\left(S_{u}^{\prime} \times S_{v}^{\prime}\right) \cdot n\right) \arccos \frac{F}{\sqrt{E G}}
$$

Let $\varphi_{a u}$ and $\varphi_{a v}$ be the angle of any tangent vector $\delta S$ from $S_{u}^{\prime}$ and $S_{v}^{\prime}$, and the counter-clockwise direction is defined as positive. If $\delta S=S_{u}^{\prime} \delta u+S_{v}^{\prime} \delta v$, we obtain

$$
\begin{aligned}
& S_{u}^{\prime} \cdot \delta S=E \delta u+F \delta v \\
& S_{v}^{\prime} \cdot \delta S=F \delta u+G \delta v
\end{aligned}
$$

and

$$
\|\delta S\|=\sqrt{\delta S \cdot \delta S}=\sqrt{E(\delta u)^{2}+2 F \delta u \delta v+G(\delta v)^{2}}
$$

Therefore,

$$
\begin{gathered}
\varphi_{a u}= \pm \arccos \frac{E \delta u / \delta v+F}{\sqrt{E^{2}(\delta u / \delta v)^{2}+2 E F \delta u / \delta v+E G}} \\
\varphi_{a v}= \pm \arccos \frac{E \delta u / \delta v+G}{\sqrt{E G(\delta u / \delta v)^{2}+2 F G \delta u / \delta v+G^{2}}}
\end{gathered}
$$

The sign of the above expressions remains to be determined. The next step is to calculate the angles of the maximum major direction $P_{s 1}$ in Fig 3 from $S_{u}^{\prime}$ and $S_{v}^{\prime}, \varphi_{1 u}$ and $\varphi_{1 v} \varphi_{1 v}$.

Based on differential geometrical theory, the maximum major direction $\left.(\delta u / \delta v)\right|_{\max }$ is determined as

$$
\left.\frac{\delta u}{\delta v}\right|_{\max }=\frac{k_{s 1} F-M}{L-k_{s 1} E}=\frac{k_{s 1} G-N}{M-k_{s 1} F}
$$

where $L, M$ and $N$ are type II basic quantities. By substituting the above formula into Eq.(9) and Eq.(10), we can obtain

$$
\begin{gathered}
\varphi_{1 u}= \pm \arccos \frac{F L-E M}{\tau_{u}} \\
\varphi_{1 v}= \pm \arccos \frac{G M-F N}{\tau_{v}}
\end{gathered}
$$

where

$$
\begin{aligned}
\tau_{u} & =\left[\left(k_{s 1} E F-E M\right)^{2}+E G\left(\mathrm{~L}-k_{s 1} E\right)^{2}+2 E F\left(k_{s 1} F-M\right)\left(\mathrm{L}-k_{s 1} E\right)\right]^{0.5} \\
\tau_{v} & =\left[E G\left(k_{s 1} G-N\right)^{2}+\left(E M-k_{s 1} F G\right)^{2}+2 F G\left(k_{s 1} G-N\right)\left(\mathrm{M}-k_{s 1} F\right)\right]^{0.5}
\end{aligned}
$$

From the relationship of the angles, we determine the relationship among $\varphi_{v u}, \varphi_{1 u}$ and $\varphi_{1 v}$ as

$$
\varphi_{v u}=\varphi_{1 u}-\varphi_{1 v}
$$


If we substitute Eq. (11) and Eq. (12) into the right side of the above equation, four solutions of $\varphi_{v u}$ are obtained, and we choose $\varphi_{1 u}$ and $\varphi_{1 v}$, which are equal to Eq. (8), as angles of the maximum major vector $P_{s 1}$ from $S_{u}^{\prime}$ and $S_{v}^{\prime}$. We let $\varphi_{1 u}=\varphi_{1}$.

From Fig. 3, the angle of the maximum major vector $P_{s 1}$ from the $x_{\mathrm{c}}$ axis is $\varphi_{u}+\varphi_{1}$. Based on the Euler formula, the curvature of any direction, which is $\varphi(-\pi / 2<\varphi \leq \pi / 2)$ from the $x_{\mathrm{c}}$ axis at the CC point $\boldsymbol{C}$ on the surface $\boldsymbol{S}$, can be calculated as

$$
k_{s \varphi}=k_{s 1} \cos ^{2}\left[\varphi-\left(\varphi_{u}+\varphi_{1}\right)\right]+k_{s 2} \sin ^{2}\left[\varphi-\left(\varphi_{u}+\varphi_{1}\right)\right]
$$

Let

$$
\begin{gathered}
\xi_{u}=\sin \varphi_{u} \\
\xi_{1}=\sin \varphi_{1} \\
\eta_{u}=\cos \varphi_{u} \\
\eta_{1}=\cos \varphi_{1} \\
\alpha=\left(k_{s 2}-k_{s 1}\right)\left[\xi_{u} \eta_{u}\left(\eta_{1}^{2}-\xi_{1}^{2}\right)+\xi_{1} \eta_{1}\left(\eta_{u}^{2}-\xi_{u}^{2}\right)\right]
\end{gathered}
$$

We expand Eq.(14) using the trigonometric relations and obtain

$$
k_{s \varphi}=k_{s 0} \cos ^{2} \varphi+k_{s \pi / 2} \sin ^{2} \varphi-\alpha \sin 2 \varphi
$$

where

$$
\begin{aligned}
& k_{s 0}=\eta_{u}^{2}\left(k_{s 1} \eta_{1}^{2}+k_{s 2} \xi_{1}^{2}\right)+\xi_{u}^{2}\left(k_{s 1} \xi_{1}^{2}+k_{s 2} \eta_{1}^{2}\right)-2 \xi_{u} \eta_{u} \xi_{1} \eta_{1}\left(k_{s 1}-k_{s 2}\right) \\
& k_{s \pi / 2}=\eta_{u}^{2}\left(k_{s 1} \xi_{1}^{2}+k_{s 2} \eta_{1}^{2}\right)+\xi_{u}^{2}\left(k_{s 1} \eta_{1}^{2}+k_{s 2} \xi_{1}^{2}\right)-2 \xi_{u} \eta_{u} \xi_{1} \eta_{1}\left(k_{s 1}-k_{s 2}\right)
\end{aligned}
$$

We write the equation of $S^{*}$, the tangent surface of $S$ directly near the CC point, in the local coordinate system as

$$
S^{*}: Z_{\mathrm{c}}=\frac{1}{2}\left[k_{s 0} x_{\mathrm{c}}^{2}+k_{s \pi / 2} y_{\mathrm{c}}^{2}-2 \alpha x_{\mathrm{c}} y_{\mathrm{c}}\right]
$$

\section{Cutter path generation}

When planning the process path of complex surfaces, a common method is to give a specific initiation path and the original dig angle and rotation angle of the cutter and determine whether interference collision of the cutters occurs. If collisions exist, the situation is fixed by determining the step length based on the original or amended CL, finding the next CC point in the same path, and determining the bandwidth of the process and the distance between lines to find the $\mathrm{CC}$ point in the next path corresponding to the current CC point.

\subsection{Calculation of the step length}

As noted above, the cutter path can be considered as an ensemble of the discrete cutter location data, and the cutter location data include the CL point. Each CL point corresponds to a CC point, and the distance between two adjacent CC points is the step length we seek. Therefore, we discretize the CC' path $P_{i}$ first. The discretization is not arbitrary division but is based on process accuracy. For the $j^{\text {th }} \mathrm{CC}$ point vector $C_{i, j}$, (corresponding to parameter $w=w j$ ) in the $i^{\text {th }}$ current CC path $P_{i}=S(u(w), v(w))$, the value of the parameter $w_{j+1}$ of the $(j+1)^{\text {th }}$ CC point vector can be determined.

The curve $P$ curvature at $C_{i, j}$ can be expressed as

$$
k_{i, j}=\frac{\left\|S_{w}^{\prime} \times S^{\prime \prime}{ }_{w w}\right\|_{w=w_{j}}}{\left\|S_{w}^{\prime}\right\|_{w=w_{j}}^{3}}
$$

where

$$
\begin{gathered}
S_{w}^{\prime}=S_{u}^{\prime} u_{w}^{\prime}+S_{v} v_{w}^{\prime} \\
S^{\prime \prime}{ }_{w w}=\left(S^{\prime \prime}{ }_{u u} u_{w}^{\prime}+S^{\prime \prime}{ }_{u v} v_{w}^{\prime}\right) u_{w}^{\prime}+S_{u}^{\prime} u^{\prime \prime}{ }_{w w}+\left(S^{\prime \prime}{ }_{v u} u_{w}^{\prime}+S^{\prime \prime}{ }_{v v} v_{w}^{\prime}\right) v_{w}^{\prime}+S_{v} v^{\prime \prime}{ }_{w w}
\end{gathered}
$$

The calculation of the step length at the current CC point is shown in Fig. 4, where $\varepsilon$ is the process accuracy and $\triangle l$ is the step length given in document [8], which can be calculated as

$$
\triangle l=2\left[1 / k_{i, j}^{2}-\left(1 / k_{i, j}-\varepsilon\right)^{2}\right]^{0.5}
$$




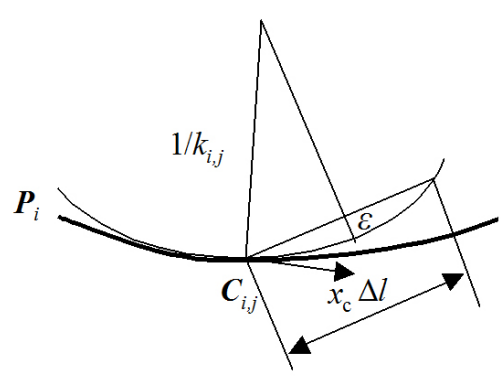

Fig 4. Calculation of the step length

After we calculate $\triangle l, w_{j+1}$ can be calculated as

$$
w_{j+1}=w_{j}+\frac{\triangle l}{\left\|S_{w}^{\prime}\right\|_{w=w_{j}}}
$$

\subsection{Calculation of the effective process bandwidth}

The effective process bandwidth $B$ refers to the distance between the process bands in the normal direction of motion. The process bandwidth describes the range limit that satisfies the accuracy requirement of the process. Exceeding this range corresponds to a deficiency in the cutting location, thus requiring additional polishing. Therefore, one of the goals of cutter path generation is to control the size of the process distance such that the final distance does not exceed the process bandwidth.

Based on the tangent surface $\boldsymbol{S}^{*}$ as depicted in Eq.(16), we can calculate the normal vector of $\boldsymbol{S}^{*}$ at any point $\left(x_{\mathrm{c}}, y_{\mathrm{c}}\right)$ in the coordinate plane $x_{\mathrm{c}} \mathrm{C} y_{\mathrm{c}}$ as

$$
\boldsymbol{n}_{s^{*}}\left(x_{\mathrm{c}}, y_{\mathrm{c}}\right)=\left[\begin{array}{c}
n_{s^{*} x} \\
n_{s^{*} y} \\
n_{s^{*} z}
\end{array}\right]=\left[\begin{array}{c}
\alpha y_{\mathrm{c}}-k_{s 0} x_{\mathrm{c}} \\
\alpha x_{\mathrm{c}}-k_{s \pi / 2} y_{\mathrm{c}} \\
1
\end{array}\right]
$$

According to the above equation, the normal vector $\boldsymbol{n}_{s^{*}}$ of $\boldsymbol{S}^{*}$ is oriented towards $\boldsymbol{\Sigma}^{*}$, the tangent surface of the cutter surface. Assuming that the allowed error is $\delta$ (the maximum of the allowed scallop height), we can write the iso-plane equation of $\boldsymbol{S}^{*}$ as

$$
\left\{\begin{array}{c}
X=x_{\mathrm{c}}+\frac{\delta n_{s^{*} x}}{\left\|\boldsymbol{n}_{s^{*}}\left(x_{\mathrm{c}}, y_{\mathrm{c}}\right)\right\|} \\
Y=y_{\mathrm{c}}+\frac{\delta n_{s^{*} y}}{\left\|\boldsymbol{n}_{s^{*}}\left(x_{\mathrm{c}}, y_{\mathrm{c}}\right)\right\|} \\
Z=z_{\mathrm{c}}+\frac{\delta n_{s^{*} z}}{\left\|\boldsymbol{n}_{s^{*}}\left(x_{\mathrm{c}}, y_{\mathrm{c}}\right)\right\|}
\end{array}\right.
$$

where $z_{\mathrm{c}}$ is determined by Eq. (16) and $X, Y$, and $Z$ are the coordinates of the points in the iso-plane of $\boldsymbol{S}^{*}$ in the local coordinate system.

Because $S^{*}$ and $\Sigma^{*}$ are tangent at the origin of the local coordinate system and $\Sigma^{*}$ is located above $S^{*}$, the iso-planes of $S^{*}$ must intersect with $\Sigma^{*}$. If the equations of the intersected lines are known, the effective bands and their width can be determined.

The above method can supply an accurate solution, but the calculation is highly complex. Because the process bandwidth B is defined in the normal direction of motion, we adopt the following approximation for the calculation of process bandwidth in this paper.

Figure 5 shows the coordinate plane $y_{\mathrm{c}} C z_{\mathrm{c}}$ of the local coordinate system. The dotted lines are the intersecting lines of the iso-planes of $\boldsymbol{S}^{*}$ and the plane $y_{\mathrm{c}} C z_{\mathrm{c}}$, and the solid lines are the intersecting lines of $\Sigma^{*}, \boldsymbol{S}^{*}$ and the plane $y_{\mathrm{c}} C z_{\mathrm{c}}$. 
Letting $x_{\mathrm{c}}=0$ in Eq. (6) and Eq. (16), we can obtain the following intersecting line equation of $\boldsymbol{\Sigma}^{*}$ and $\boldsymbol{S}^{*}$ :

$$
\begin{aligned}
& \Sigma^{*}: z_{\mathrm{c}}=k_{\Sigma \pi / 2} y_{\mathrm{c}}^{2} / 2 \\
& S^{*}: z_{\mathrm{c}}=k_{\mathrm{S} \pi / 2} y_{\mathrm{c}}^{2} / 2
\end{aligned}
$$

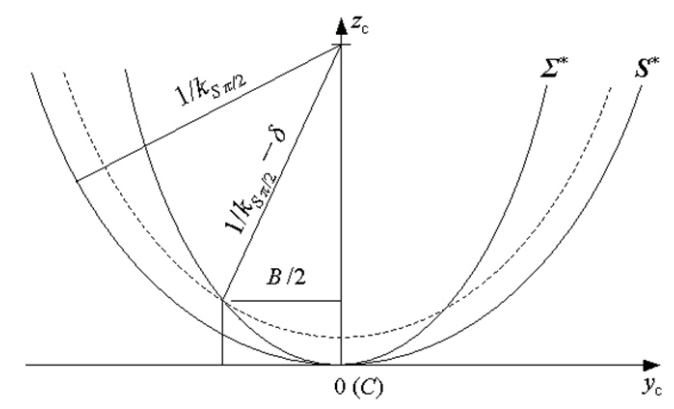

Fig 5. Model for calculating the process bandwidth

Both intersecting lines are parabolas. If $k_{\mathrm{S} \pi / 2} \neq 0$, then Eq.(20) is approximately a circle near the CC point, and the equation of the dotted lines is

$$
\left(Z_{\mathrm{c}}-1 / k_{\mathrm{S} \pi / 2}\right)^{2}+y_{\mathrm{c}}^{2}=\left(1 / k_{\mathrm{S} \pi / 2}-\delta\right)^{2} \quad k_{\mathrm{S} \pi / 2} \neq 0
$$

By combining Eq.(19) and Eq.(21), we can calculate the coordinate of the intersecting point $y_{\mathrm{c}}$ of the dotted lines and $\Sigma^{*}$ as

$$
y_{\mathrm{c}}= \pm\left\{\frac{2 \operatorname{sgn}\left(k_{\mathrm{S} \pi / 2}\right)}{k_{\Sigma \pi / 2}}\left[\left(\frac{1}{k_{S \pi / 2}}-\frac{1}{k_{\Sigma \pi / 2}}\right)^{2}-\frac{2 \delta}{k_{S \pi / 2}}+\delta^{2}\right]^{0.5}+\frac{2}{k_{\Sigma \pi / 2}}-\frac{2}{k_{S \pi / 2} k_{\Sigma \pi / 2}}\right\}^{0.5} \quad k_{\mathrm{S} \pi / 2} \neq 0
$$

If $k_{\mathrm{S} \pi / 2}=0$, based on Eq.(20), the intersecting line of $\boldsymbol{S}^{*}$ is the $y_{\mathrm{c}}$ axis, and we obtain the following expression:

$$
y_{\mathrm{c}}= \pm \sqrt{\frac{2}{k_{\Sigma \pi / 2}}} \quad k_{\mathrm{S} \pi / 2}=0
$$

Based on the symmetry about $z_{\mathrm{c}}$, the process bandwidth $\mathrm{B}$ corresponding to the $\mathrm{CC}$ point $C$ can be calculated as

$$
B=2\left|y_{\mathrm{c}}\right|
$$

\subsection{Calculation of the process distance}

The process distance is the distance between the corresponding CC points of two adjacent CC paths. The process distance is typically a function of the allowed scallop height $\delta$, the effective cutting radius and the radius of normal curvature of the surface in the direction of the distance. The direction of the distance is the normal direction of the current path. In this direction, the effective cutting radius can be expressed as

$$
r_{\mathrm{e}}=1 / k_{\Sigma \pi / 2}=\frac{r_{\mathrm{f}}}{1-\frac{r \cos ^{2} \omega}{r+r_{\mathrm{f}} \sin \lambda}}
$$

The normal curvature radius of the processed surface is

$$
r_{\mathrm{b}}=1 /\left|k_{S \pi / 2}\right|
$$

The surface and cutter are typically approximated as circles after $r_{\mathrm{e}}$ and $r_{\mathrm{b}}$ are calculated, and the distance $D_{i, j}$ is calculated as

$$
D_{i, j}=\sqrt{\frac{8 r_{\mathrm{b}} r_{\mathrm{e}} \delta}{r_{\mathrm{b}}+r_{\mathrm{e}} \operatorname{sgn}\left(k_{S \pi / 2}\right)}}
$$

A better formula to calculate the distance can be established based on the research on process bandwidth mentioned 
above. To improve the process efficiency, we set the distance as the process bandwidth of the current CC path. Therefore, we can calculate the distance as

$$
D_{i, j}= \begin{cases}\sqrt{8 r_{\mathrm{e}} \operatorname{sgn}\left(k_{S \pi / 2}\right) \sqrt{\left(\frac{1}{k_{S \pi / 2}}-r_{\mathrm{e}}\right)^{2}-\frac{2 \delta}{k_{S \pi / 2}}+\delta^{2}}+8 r_{\mathrm{e}}-\frac{8 r_{\mathrm{e}}}{k_{S \pi / 2}}} & k_{\mathrm{S} \pi / 2} \neq 0 \\ \sqrt{8 r_{\mathrm{e}} \delta} & k_{\mathrm{S} \pi / 2}=0\end{cases}
$$

\subsection{Calculation of the adjacent $\mathrm{CC}$ points}

As stated above, the current path is the $i^{\text {th }}$ CC point path $\boldsymbol{P}_{i}=\boldsymbol{S}(u(w), v(w))$ on the parameter surface $S=S(u, v)$, the current CC point is $C_{i, j}=\boldsymbol{S}\left(u\left(w_{j}\right), v\left(w_{j}\right)\right)$, and the next path and its CC point are $\boldsymbol{P}_{i+1}$ and $\boldsymbol{C}_{i+1, j}$, respectively. Assuming that $\boldsymbol{C}_{i+1, j}$ is oriented in the normal direction of the current motion direction, i.e., the direction of distance, we obtain

$$
\left(\boldsymbol{C}_{i+1, j}-\boldsymbol{C}_{i, j}\right) \cdot \boldsymbol{S}_{w}\left(u\left(w_{j}\right), v\left(w_{j}\right)\right)=0
$$

which can be reformatted as

$$
\left(\boldsymbol{C}_{i+1, j}-\boldsymbol{C}_{i, j}\right) \cdot\left(\boldsymbol{S}_{u}^{\prime} u_{w}^{\prime}+\boldsymbol{S}_{v}^{\prime} v_{w}^{\prime}\right)_{w=w_{j}}=0
$$

Based on the process distance, one obtains

$$
\boldsymbol{D}_{i, j}=\left\|\boldsymbol{C}_{i+1, j}-\boldsymbol{C}_{i, j}\right\|
$$

We can use the Taylor formula to expand $\boldsymbol{C}_{i+1, j}$ at $\boldsymbol{C}_{i, j}$ and ignore the $2^{\text {nd }}$ - and higher-order residuals:

$$
\boldsymbol{C}_{i+1, j}=\boldsymbol{C}_{i, j}+\boldsymbol{S}_{u}^{\prime} \triangle u+\boldsymbol{S}_{v}^{\prime} \triangle v
$$

By substituting this equation into Eq.(25) and Eq.(26), we obtain

$$
\begin{aligned}
& \left(E u_{w}^{\prime}+F v_{w}^{\prime}\right) \triangle u+\left(F u_{w}^{\prime}+G v_{w}^{\prime}\right) \triangle v=0 \\
& E(\triangle u)^{2}+2 F(\triangle u \triangle v)+G(\triangle v)^{2}=D_{i, j}^{2}
\end{aligned}
$$

Combining the two equations above yields

$$
\left\{\begin{array}{l}
\triangle u= \pm \frac{\alpha_{2} D_{i, j}}{\sqrt{E \alpha_{2}^{2}+2 F \alpha_{1} \alpha_{2}+G \alpha_{1}^{2}}} \\
\triangle v= \pm \frac{D_{i, j}}{\sqrt{E \frac{\alpha_{2}^{2}}{\alpha_{1}^{2}}+2 F \frac{\alpha_{2}}{\alpha_{1}}+G}}
\end{array}\right.
$$

where the sign is determined by the actual process direction and

$$
\begin{gathered}
\alpha_{1}=E u_{w}^{\prime}+F v_{w}^{\prime} \\
\alpha_{2}=-\left(F u_{w}^{\prime}+G v_{w}^{\prime}\right)
\end{gathered}
$$

By applying Eq.(27), the current CC point's adjacent CC point

$$
\boldsymbol{C}_{i+1, j}=\boldsymbol{S}\left(u\left(w_{j}\right)+\Delta u, v\left(w_{j}\right)+\Delta v\right)
$$

\subsection{From the CC point to the $C L$ point}

As shown in Fig. 2, the CL point vector $\boldsymbol{O}$ and the unit vector of cutter axis $\boldsymbol{T}$ can be expressed in the cutter coordinate system as

$$
\begin{aligned}
& \boldsymbol{O}_{\mathrm{t}}=\left[\begin{array}{llll}
0 & 0 & 0 & 1
\end{array}\right]^{\mathrm{T}} \\
& \boldsymbol{T}_{\mathrm{t}}=\left[\begin{array}{llll}
0 & 0 & 1 & 0
\end{array}\right]^{\mathrm{T}}
\end{aligned}
$$

After the coordinate transformation, the CL point vector and the unit vector of cutter axis in the local coordinate system 
can be obtained as

$$
\boldsymbol{O}_{\mathrm{c}}=\left[\begin{array}{c}
-\left(r \cos \lambda+r_{\mathrm{f}} \sin \lambda\right) \cos \omega \\
-\left(r \cos \lambda+r_{\mathrm{f}} \sin \lambda\right) \sin \omega \\
r \sin \lambda-r_{\mathrm{f}} \cos \lambda \\
1
\end{array}\right] \quad \boldsymbol{T}_{\mathrm{c}}=\left[\begin{array}{c}
\sin \lambda \cos \omega \\
\sin \lambda \sin \omega \\
\cos \lambda \\
0
\end{array}\right]
$$

We assume that the unit sectors of the $x_{\mathrm{c}}, y_{\mathrm{c}}$ and $z_{\mathrm{c}}$ axes of the local coordinate system are $\boldsymbol{i}_{\mathrm{c}} 、 \boldsymbol{j}_{\mathrm{c}}$ and $\boldsymbol{k}_{\mathrm{c}}$ in the object coordinate system, respectively, where

$$
\begin{gathered}
\boldsymbol{i}_{\mathrm{c}}=\boldsymbol{S}_{w}^{\prime} /\left\|\boldsymbol{S}_{w}\right\| \\
\boldsymbol{j}_{\mathrm{c}}=\boldsymbol{k}_{\mathrm{c}} \times \boldsymbol{i}_{\mathrm{c}} \\
\boldsymbol{k}_{\mathrm{c}}=\boldsymbol{S}_{u}^{\prime} \times \boldsymbol{S}_{v}^{\prime} /\left\|\boldsymbol{S}_{u}^{\prime} \times \boldsymbol{S}_{v}^{\prime}\right\|
\end{gathered}
$$

and the origin $C$ of the local coordinate system is $C_{i, j}$ in the object coordinate system. Therefore, the transformation matrix from the local coordinate system to the object coordinate system is written as

$$
\boldsymbol{M}=\left[\begin{array}{cccc}
\boldsymbol{i}_{\mathrm{c}} & \boldsymbol{j}_{\mathrm{c}} & \boldsymbol{k}_{\mathrm{c}} & \mathrm{C}_{i, j} \\
0 & 0 & 0 & 1
\end{array}\right]
$$

Therefore, the CL data in the object coordinate system is expressed as

$$
\left\{\begin{array}{l}
\boldsymbol{O}_{w}=\boldsymbol{M} \boldsymbol{O}_{\mathrm{c}} \\
\boldsymbol{T}_{w}=\boldsymbol{M} \boldsymbol{T}_{\mathrm{c}}
\end{array}\right.
$$

\section{Inspection of local interference and correction of the cutter posture}

In the above discussion, we assume that the position angles of the cutters (the dip angle $\lambda$ and rotation angle $\omega$ ) are the angles after local inspection. In real work, we must make a correction to a possible unreasonable cutter posture before calculating the cutter path. However, many combinations can satisfy the non-local-interference requirement, but certain combinations generate a large cutting bandwidth, whereas others generate a small cutting bandwidth. Therefore, the posture of the cutters must be controlled from the perspective of disposing of more materials. The purpose of this section is to establish a method that meets the non-local-interference requirement and can remove more materials.

\subsection{Effect of the dip angle and rotation angle on the process result}

In Fig $6 \mathrm{a}$, the rotation angle $\omega$ is the same and $\lambda_{1}>\lambda_{2}$. From the formula of the effective cutting radius, $r_{\mathrm{e} 1}<\lambda_{\mathrm{e} 2}$, and with the same requirement as the scallop height, the process bandwidth $B_{1}<B_{2}$. This information indicates that a larger dip angle results in a smaller process bandwidth, which decreases the efficiency of the process. Therefore, the cutter should not be tilted if there is no interference.

In Fig. 6b, the dip angle $\lambda$ is the same and $\left|\omega_{1}\right|>\left|\omega_{2}\right|$, and thus, we have a similar conclusion. Therefore, under the condition of the same accuracy and interference, the cutter should not be rotated.

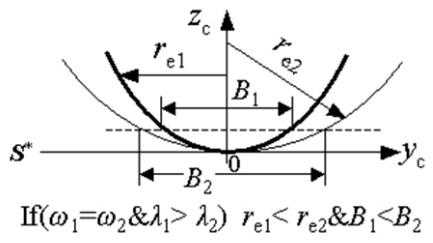

(a)

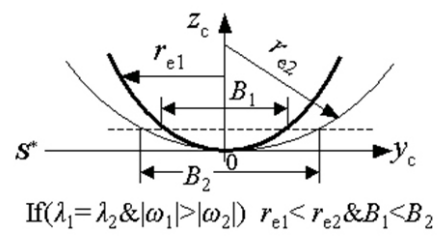

(b)

Fig 6. Effect of the dip angle and rotation angle on the process result

To summarize the analysis of the effect of dip angle and rotation angle on the result of the process, we conclude the following for the determination of the original posture of the cutters: (i) The original rotation angle $\omega_{0}$ should be set to $0^{\circ}$. (ii) Because of the local convex differential geometrical structure and considering the follow-up interference even though the local interference is unlikely at flat CC points, the original dip angle $\lambda_{0}$ can be set to range from $2^{\circ}$ to $5^{\circ}$. (iii) 
For other types of points, such as concave points or hyperbolic points, the angles can be determined using the original angle formula as

$$
\lambda_{0}=\arcsin \left[\left(r+r_{\mathrm{f}}\right)\left|k_{S \pi / 2}\right|\right]+2^{\circ} \sim 5^{\circ}
$$

\subsection{Optimization of the dip angle and rotation angle}

Local interference of CC points occurs when the effective curvature of the cutter surface $\Sigma$ is smaller than the effective curvature of the object surface $S$ in a certain direction. To avoid local interference, we should ensure that the effective curvature of the cutter is no less than the effective curvature of the object surface in all directions, i.e.,

$$
k_{\Sigma \varphi} \geq k_{S \varphi} \quad \forall \varphi \in(-\pi / 2, \pi / 2)
$$

In the above expression, $k_{\Sigma \varphi}$ and $k_{S \varphi}$ are taken from Eq.(5) and Eq.(15), respectively; $k_{\Sigma \varphi}$ is relevant to the size of the cutter, the dip angle $\lambda$ and the rotation angle $\omega$, etc.; and $k_{S \varphi}$ is relevant to the motion direction and the shape of the surface near the CC point. Given that the moving direction is constant, in the expression of $k_{S \varphi}$, the parameters $k_{S 1}$, $k_{S 2}$, $k_{S 0}, k_{S \pi / 2}$ and $\alpha$ are all constants. Therefore, $k_{\Sigma \varphi}$ and $k_{S \varphi}$ are functions of $\varphi$ with a given cutter, motion direction, dip angle $\lambda$ and rotation angle $\omega$, and their difference is also a function of $\varphi$

$$
f(\varphi)=k_{\Sigma \varphi}-k_{S \varphi} \quad \varphi \in(-\pi / 2, \pi / 2)
$$

Substituting Eq.(5) and Eq.(15) into the above expression, we obtain the following

$$
f(\varphi)=\left(k_{\Sigma 0}-k_{S 0}\right) \cos ^{2} \varphi+\left(k_{\Sigma \pi / 2}-k_{S \pi / 2}\right) \sin ^{2} \varphi+\left[\frac{1}{2}\left(k_{\Sigma 1}-k_{\Sigma 2}\right) \sin 2 \omega+\alpha\right] \sin 2 \varphi
$$

The first derivative of $f(\varphi)$ is written as follows

$$
f^{\prime}(\varphi)=b \sin 2 \varphi+c \cos 2 \varphi=\sqrt{b^{2}+c^{2}} \sin (2 \varphi+\theta)
$$

where $b=k_{\Sigma \pi / 2}-k_{S \pi / 2}+k_{S 0}-k_{\Sigma 0}, c=2 \alpha+\left(k_{\Sigma 1}-k_{\Sigma 2}\right) \sin 2 \omega$, the auxiliary angle $\theta$ and $(b, c)$ are in the same quadrant, and $\tan \theta=c / b$. Taking the value range of $\varphi$ considered, $\theta$ can be determined by the following equation (generally $b$ and $c$ are not zero simultaneously).

$$
\theta= \begin{cases}\arctan (c / b) & b \geq 0 \\ \arctan (c / b)+\pi & b<0 \cap c \geq 0 \\ \arctan (c / b)-\pi & b<0 \cap c<0\end{cases}
$$

Letting $f^{\prime}(\varphi)=0$, we calculate the stagnation point of $f(\varphi)$ as

$$
\varphi_{\text {stagnation }}=(n \pi-\theta) / 2
$$

Because $\theta \in(-\pi, \pi]$ if and only if $n=0, \varphi_{\text {stagnation }}(-\pi / 2, \pi / 2]$, we obtain

$$
\varphi_{\text {stagnation }}=-\theta / 2
$$

We calculate the second derivative of $f(\varphi)$ as

$$
f^{\prime \prime}(\varphi)=2 \sqrt{b^{2}+c^{2}} \cos (2 \varphi+\theta)
$$

Substituting Eq.(33) into Eq.(34) yields $f^{\prime \prime}\left(\varphi_{\text {stagnation }}\right)>0$; therefore, the stagnation point $\varphi=\varphi_{\text {stagnation }}$ is a minimum point of $f(\varphi)$.

To ensure that Eq. (30) applies, we need $f(\varphi) \geq 0$. With awareness of the continuation of $f(\varphi)$ in the interval of $\varphi$ and the only minimum point of $f(\varphi)$ in the interval, the condition of non-local-interference is

$$
f\left(\varphi_{\text {stagnation }}\right) \geq 0
$$

Substituting Eq.(33) into the above expression yields

$$
\left(k_{\Sigma 0}-k_{S 0}\right) \cos ^{2} \frac{\theta}{2}+\left(k_{\Sigma \pi / 2}-k_{S \pi / 2}\right) \sin ^{2} \frac{\theta}{2}-2 c \sin \theta \geq 0
$$

After simplification,

$$
\mathrm{d}-b \cos \theta-4 \mathrm{c} \sin \theta \geq 0
$$


where $d=k_{\Sigma \pi / 2}-k_{S \pi / 2}+k_{\Sigma 0}-k_{S 0}$.

Because $\tan \theta=c / b$, Eq.(35) can be converted into Eq.(36):

$$
d \sqrt{b^{2}+c^{2}} \geq b^{2}+4 c^{2}
$$

With the given cutter and motion direction, $b, c$ and $d$ in the above expression are functions of the dip angle $\lambda$ and rotation angle $\omega$.

\subsection{Summary of local interference inspection and correction}

The process of local interference inspection and correction is described as follows: (1) Calculate the original angle $\lambda_{0}$ and $\omega_{0}$. (ii) Verify whether $\lambda$ and $\omega$ satisfy Eq.(36). (iii) If they do, the process returns to the calculation of the adjacent CC point and CL data. (iv) If no, verify whether $\lambda$ is larger than $45^{\circ}$; if not, $\omega$ is unchanged, $\lambda$ is assigned a preset increment $\Delta \lambda$, and the process returns to (ii); if yes, $\lambda$ is unchanged and $\omega$ is assigned a preset increment $\Delta \omega$, and the process returns to (ii).

\section{Process of cutter path generation}

First, we determine an initial process $\mathrm{CC}$ path, calculate the current $\mathrm{CC}$ point for the path and calculate the relevant local data of the object surface using the method described in Section 2.2. Second, we determine the original angles of the cutter posture based on (30) and calculate the relevant local data of the cutter surface with the selected angles. We determine whether (36) holds; if it does not hold, we optimize the angles using the method in Section 4.3, and if it does hold, we calculate the step length, distance of lines, adjacent CC point parameters and CL data. After calculating the current $\mathrm{CC}$ path, we simulate the parameters of the adjacent CC paths. We execute the above procedure for all CC paths and output the cutter path. The cutter path generation process is summarized in the flowchart shown in Fig 7.

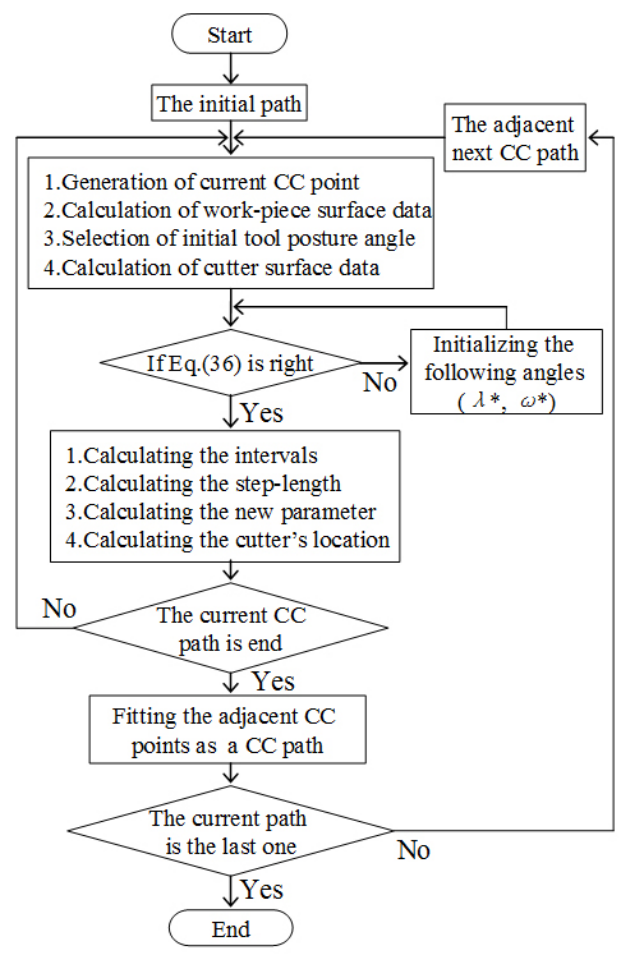

Fig 7. Flowchart of cutter path generation

\section{Example}

The processed surface has approximate dimensions of $400 \mathrm{~mm}, 200 \mathrm{~mm}$ and $50 \mathrm{~mm}$ in the $x, y$ and $z$ directions, respectively. This area consists of convex surfaces, concave surfaces and saddle surfaces. Based on the proposed method for generating cutter paths, we wrote a program in the VC environment to realize the algorithm. The process conditions 
are $r=4 \mathrm{~mm}$ and $r_{\mathrm{f}}=2 \mathrm{~mm}$. The allowable error is $\delta=0.1 \mathrm{~mm}$, and the generated CC point paths are shown in Fig $8 \mathrm{a}$.

Figure $8 \mathrm{~b}$ shows the $\mathrm{CC}$ paths generated from the traditional iso-perimetric method with the same process conditions.

Select important parameters of the two methods are compared in Table 1.

Table 1. Comparison of parameters

\begin{tabular}{cccc}
\hline Used method & Total number of the CC paths & $\begin{array}{c}\text { Total length of the CC paths } \\
L / \mathrm{mm}\end{array}$ & $\begin{array}{c}\text { Average bandwidth } \\
D / \mathrm{mm}\end{array}$ \\
\hline Proposed method & 33 & 12948 & 7.6 \\
Iso-perimetric method & 51 & 24528 & 4.9 \\
\hline
\end{tabular}

The data listed in Table 1 show that the proposed method can obtain a larger average bandwidth and a smaller number of the CC paths relative to the iso-perimetric method. At the same time, the total length of the CC paths is also shorter, which means that under the same cutting speed, the processing time of the surface will be shortened for given accuracy requirement. On the premise of ensuring the machining accuracy, the machining efficiency can be effectively improved by using the proposed method. The simulated process with the 5-axis process path from the proposed method is shown in Fig 9. Figure 9a and 9b respectively describe the situations of the initial stage of machining and at the end of machining.

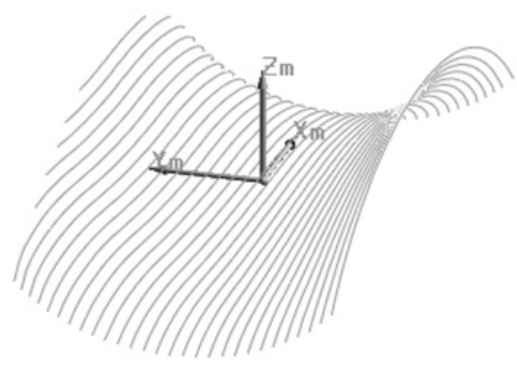

(a)

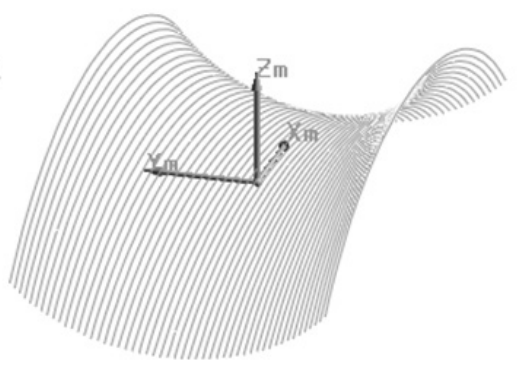

(b)

Fig 8. Paths of the CC point

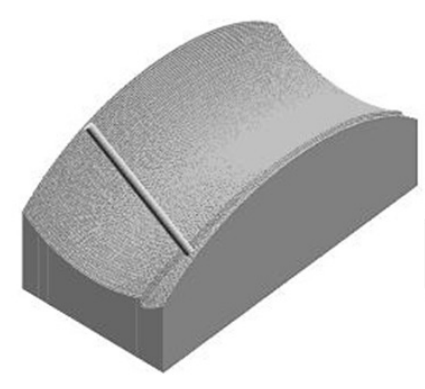

(a)

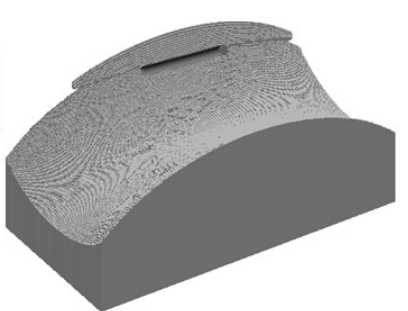

(b)

Fig 9. Simulation of machining

\section{Conclusion}

In this paper, the curvature formula in any direction of the torus-end cutter surface and object surface can be deduced using the Euler formula. Based on the above curvature formula, a new calculation method for the process distance was established. The size of the distance is closely related to the allowable process error, the curvature of the cutter surface and the object surface. The distance calculated using this method is more consistent with the real situation than those 
calculated using current methods. A larger dip angle or rotation angle results in a smaller process bandwidth and lower efficiency. Therefore, we attempt to keep the cutter straight without tilting or rotating under the pre-condition of no interference. By calculating the first and second derivatives of the condition function to avoid local interference, the most likely position of interference is found to be the position of the stagnation point. Therefore, we can check whether interference exists at the stagnation point only in the cutter interference inspection.

The above research results of this paper are concluded using a torus-end cutter, however, they are not suitable for flatend or ball-end cutters. How to use the differential geometry theory to get the unified tool path generation method will be the future work of this paper.

\section{References}

[1] He, Ying. Chen, Zhitong and Wu, Xianzhen (2014). Iso-parametric tool path overlapping method for sculptured surfaces in wide strip machining, Acta Aeronautica et Astronautica Sinica, vol. 35, no. 4. pp 1142-1148.

[2] Ding, S. Manan M. A. Poo A. N. et al.(2003). Adaptive iso-planar tool path generation for machining of free-form surfaces, Computer-Aided Design, vol.35. pp 141-153.

[3] Duvedi, Ravinder Kumar. Batish, Ajay.Bedi, Sanjeev and Mann, Stephen(2015). Scallop height of 5-axis machining of large triangles with a flat end mill, Computer-Aided Design and Applications, vol.12, no.6. pp710-716.

[4] Grandguillaume, Laureen. Lavernhe, Sylvain and Tournier, Christophe(2016).A tool path patching strategy around singular point in 5-axis ball-end milling, International Journal of Production Research, vol.54, no. 24. pp 74807490 .

[5] Yigit, I.E. Layegh K. S. Ehsan and Lazoglu, Ismail(2015). A solid modeler based engagement model for 5-axis ball end milling, Procedia CIRP, vol. 31. pp179-184.

[6] Sun, Chao and Altintas, Yusuf (2016). Chatter free tool orientations in 5-axis ball-end milling, International Journal of Machine Tools and Manufacture, vol. 106. pp 89-97.

[7] Dong, Zuomin and Jun, Martin B.G.(2017). Optimal tool orientation generation and chip volume/cutting force predictions for 5-axis CNC machining of curved surfaces using flat-end mills, Computer-Aided Design and Applications, Vol.14, no.3. pp 331-342.

[8] Sekine, Tsutomu and Obikawa, Toshiyuki(2015).Novel path interval formulas in 5-axis flat end milling, Applied Mathematical Modelling, vol. 39, no. 12. pp 3459-3480.

[9] Fan, Jianhua and Ball, Alan(2014). Flat-end cutter orientation on a quadric in five-axis machining, Computer Aided Design, vol. 53. pp 126-138.

[10] Ji, Wei. Liu, Xianli. Wang, Lihui and Wang, Guangyue(2016). Research on modelling of ball-nosed end mill with chamfered cutting edge for 5-axis grinding, International Journal of Advanced Manufacturing Technology, vol. 87, no. 9-12. pp 2731-2744.

[11] Konobrytskyi, Dmytro. Hossain, Mohammad M. Tucker, Thomas M. Tarbutton, Joshua A. and Kurfess, Thomas R.(2018). 5-Axis tool path planning based on highly parallel discrete volumetric geometry representation: Part I contact point generation, Computer-Aided Design and Applications, vol. 15, no. 1. pp 76-89.

[12] Sun, Yuwen. Xu, Jinting. Jin, Chunning and Guo, Dongming(2016). Smooth tool path generation for 5-axis machining of triangular mesh surface with nonzero genus, Computer Aided Design, vol. 79. pp 60-74. 